\title{
Choroidal metastases of thymic carcinoma in ultrasonographic imaging and optical coherence tomography
}

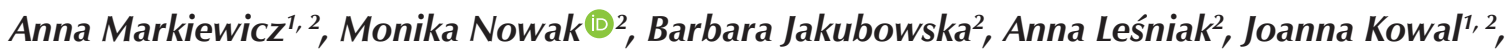 \\ Magdalena Dębicka-Kumela', ${ }^{1,}$, Bożena Romanowska-Dixon ${ }^{1,2}$
}

${ }^{1}$ Department of Ophthalmology and Ocular Oncology, Faculty of Medicine, Jagiellonian University Medical College, Krakow, Poland ${ }^{2}$ Department of Ophthalmology and Ocular Oncology, University Hospital, Krakow, Poland

Key words: thymic carcinoma; choroid metastases; ocular metastases

Thymic carcinoma is a very rare malignant mediastinal tumour with a poor prognosis. The differential diagnosis, based on histological, immunohistochemical, and genetic criteria, must also take into consideration the thymoma and thymic carcinoid as well as the metastases from the primary focus in other locations - most frequently lung cancer. Thymic carcinoma displays local malignancy, infiltrating the pleural cavity, bones, and liver. The basic treatment method in such cases is surgical resection of the involved organ, in some cases with adjuvant radiotherapy or chemotherapy. Patients with generalised disease are qualified to systemic treatment, which is most frequently multidrug chemotherapy $[1,2]$. We present a unique case of choroidal metastases of thymic carcinoma with the presentation of its ultrasound imagining and optical coherence tomography.

This female patient was aged 58 years, without a history of ophthalmological treatment, and was referred in July 2019 to the Ophthalmological Oncology Outpatient Clinic with a suspicion of an intraocular tumour of the right eye. The patient's complaint concerned a significant deterioration of vision in the right eye for about 2 months, which was preceded by the presence of flashes and floaters within the field of vision. Her medical history revealed a squamous cell thymic carcinoma in the dissemination phase in her chest. Between January 2015 and October 2016 the patient was treated with palliative chemotherapy, with adjuvant radiotherapy in April 2017. From 2018 there was a documented progression of the lesions within the chest.

In the first ophthalmological assessment performed in our centre, BCVA of the right eye was 20/400, of the left eye $-20 / 20$, whilst intraocular pressure was 16 and $18 \mathrm{mmHg}$, respectively.

The anterior segment of both eyes was unremarkable. Indirect ophthalmoscopy of the left eye fundus revealed no abnormalities, whereas the fundus of the right eye revealed a yellowish, elevated area. The picture of the lesion was suggestive of a metastasis [3, 4] (Fig. 1).

In the ultrasound (USG) optical coherence tomography (DRI-OCT) of the tumour in the right eye, some characteristic properties of metastatic tumours were observed (Fig. 1 and 2) [3, 4]. In the ultrasound, the

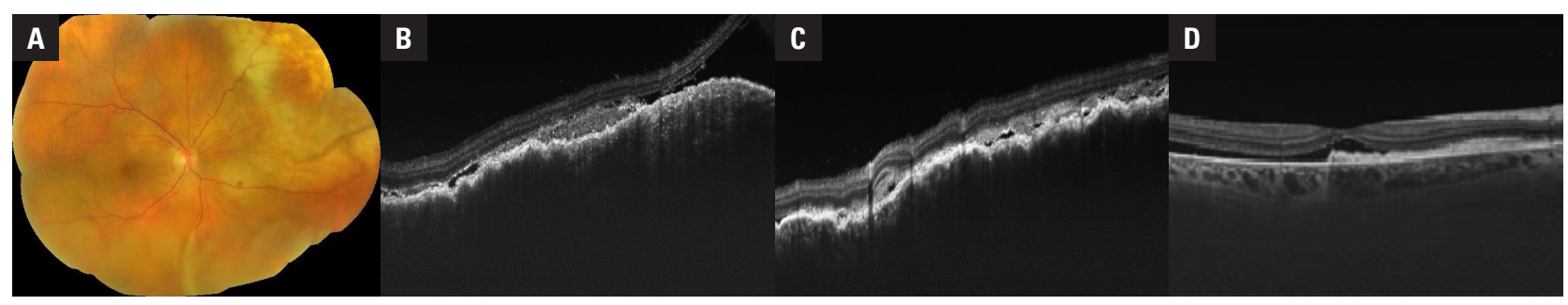

Figure 1. Clinical picture of the choroidal metastases of thymic carcinoma with accompanying serous retinal detachment as of 27 July 2018 (A). The DRI-OCT of the lesion reveals an uneven surface of the lesion, which is characteristic of metastatic tumours as well as other features of metastatic lesions, such as choriocapillaris pressure through the tumour, oedema of the photoreceptor layer, and the presence of fluid and concrements underneath the neurosensory retina (B, C). Similar exudative lesions were observed in the DRI-OCT examination of the area of the macula (D) 


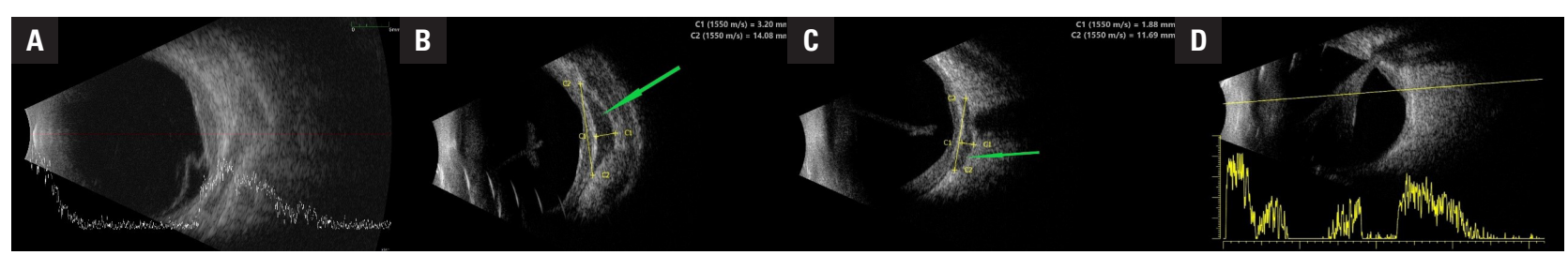

Figure 2. Ultrasound (USG) of the right eye during the first visit (25.07.2019) revealed a lesion located in the nasal and upper nasal side in the choroid, which had changeable reactivity and irregular internal structure, as well as retinal detachment located inferiorly (A). CT scan from 11.03.2020 revealed a visible extraocular infiltration (marked with green arrow) in the location corresponding to the metastatic tumour located in the choroid (B, C) and conical-shaped retinal detachment (D)

largest diameter of the tumour base was $14.05 \mathrm{~mm}$, and a thickness of $2.14 \mathrm{~mm}$ (without the eyeball wall). Additionally, the presence the retinal detachment in the inferior part was confirmed in ultrasound and exudative lesions in the area of the optic disc in DRI-OCT.

The patient was qualified for the Ru-106 brachytherapy of the right eye. On account of the poor condition of her health on the day of the hospital admission (August 2019), the procedure was abandoned. A decision was made to perform transpupillary thermotherapy of the tumour, obtaining oedema and whitening of the lesion. In March 2020, during the last follow-up visit, visual acuity in the right eye deteriorated to the light perception without projection. The examination revealed completely blister-like retinal detachments, which also made an assessment of the fundus of the affected eye completely impossible. The ultrasound revealed a hypogenic structure, not described previously and located outside the eyeball, connected to its wall and corresponding to extraocular infiltration [3]. In the left eye, during the entire follow-up period, no lesions suspected of being cancerous were found. Our patient died a few months after the last follow-up visit.

Choroidal cancer metastases are the most frequent intraocular eyeball cancers. The lesions are usually numerous, slightly elevated light-coloured changes in the posterior pole of the eye, frequently accompanied by exudative retinal detachment, and often they appear in both eyes. The cancer, which most frequently metastasises into the choroid is breast cancer, followed by lung cancer [3-5]. However, it must be remembered that rare cancers may also develop metastases into the choroid, and these may pose a significant challenge. That is why we have decided to discuss the case of choroid metastases of thymic carcinoma. Primary thymic cancers react poorly to currently available treatment, and the presence of distant metastases significantly worsens the prognoses of these patients [1,2]. There are published reports about the metastases of this cancer into the choroid, yet none of these reports discuss the fast and non-invasive imaging diagnostics with the use of OCT and USG. Moreover, during the clinical observation a tendency to form an extraocular infiltration of the metastases originating from the primary thymic cancer were found in spite of the small size of the intraocular tumour. To our knowledge, this is the first case described in the English-language literature on the subject, which might be indicative of the high aggressiveness of the thymic carcinoma and its metastases.

\section{Disclosure statement}

The authors have no conflicts of interest to disclose.

\section{Statements of ethics}

The research was conducted ethically in accordance with the World Medical Association Declaration of Helsinki. Data were collected retrospectively.

\section{Funding}

No funding was available.

\section{Competing interests}

None declared.

\section{References}

1. Ettinger D, Riely G, Akerley W, et al. Thymomas and Thymic Carcinomas: Clinical Practice Guidelines in Oncology. J Natl Compr Canc Netw. 2013; 11(5): 562-576, doi: 10.6004/jnccn.2013.0072, indexed in Pubmed: 23667206.

2. Scorsetti M, Leo F, Trama A, et al. Thymoma and thymic carcinomas. Crit Rev Oncol Hematol. 2016; 99: 332-350, doi: 10.1016/j.critrevonc.2016.01.012, indexed in Pubmed: 26818050.

3. Romanowska-Dixon B, Jakubowska B. Intraocular metastases. In: Jager Martine J. ed. Ocular Oncology. PZWL, Warszawa 2020: 317-346.

4. Mathis T, Jardel P, Loria O, et al. New concepts in the diagnosis and management of choroidal metastases. Prog Retin Eye Res. 2019; 68: 144-176, doi: 10.1016/j.preteyeres.2018.09.003, indexed in Pubmed: 30240895.

5. Shields CL, Shields JA, Gross NE, et al. Survey of 520 eyes with uveal metastases. Ophthalmology. 1997; 104(8): 1265-1276, doi: 10.1016/s0161-6420(97)30148-1, indexed in Pubmed: 9261313. 